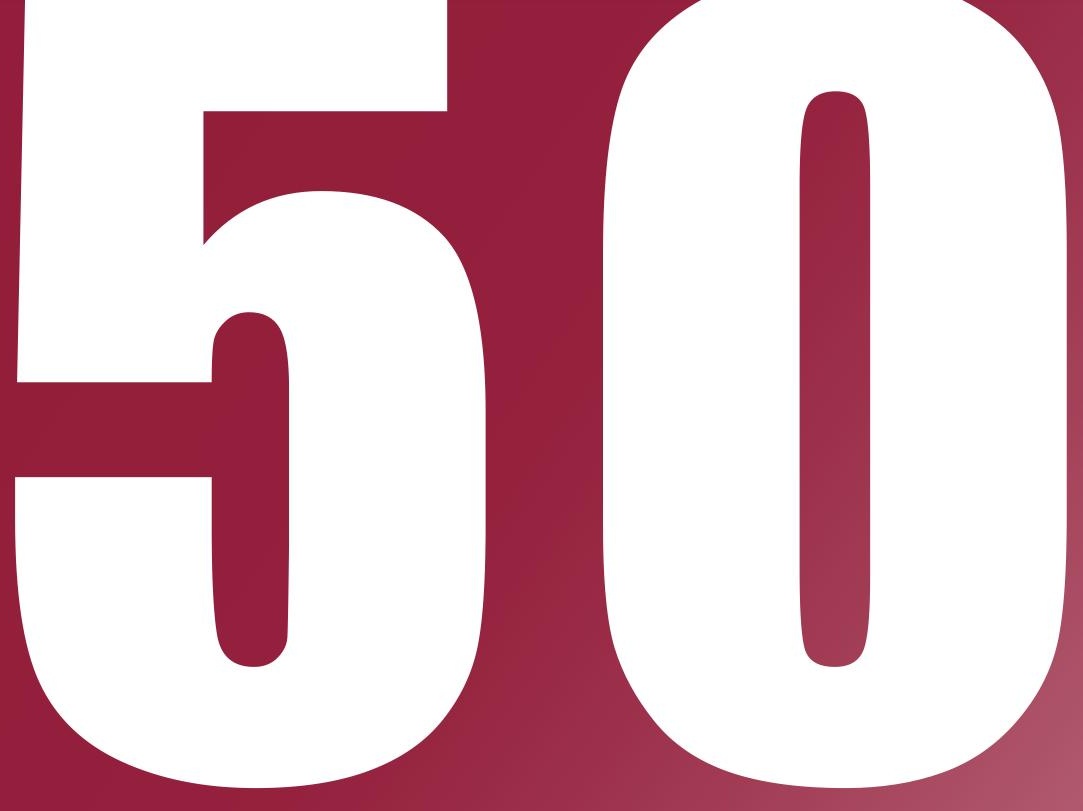

ÁMBITOS

REVISTA

INTERNACIONAL

DE

COMUNICACIÓN

$\mathbf{N}^{\circ} \mathbf{5 0}$

EDICIÓN OTOÑO

2020

ISSN: 1139-1979

E-ISSN: 1988-5733

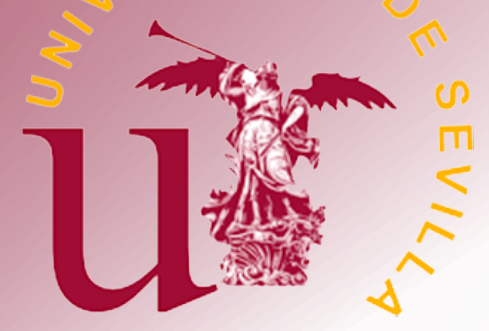




\section{INDICE}

EDITORIAL EDITORIAL

Presentación del Monográfico. Comunicación y juventud. Nuevos medios, representación, recepción y participación en contenidos de entretenimiento e información

Presentation of the monograph. Communication and youth. New media, representation, reception and participation in entertainment and information content.

Juan Francisco Gutiérrez Lozano, Francisco Javier Ruiz del Olmo

7-9

\section{MONOGRÁFICO MONOGRAPH}

El consumo audiovisual de la Generación Z. El predominio del vídeo online sobre la televisión tradicional The audiovisual consumption of Generation $Z$. The predominance of online video over traditional television

La imagen de los jóvenes en las estrategias transmedia de las series de televisión: el caso de Skam España en Instagram

The image of young people in transmedia strategies of TV series: the case of Skam España on Instagram

Sergio Jesús Villén Higueras, Francisco Javier Ruiz del Olmo

La penetración de Netflix en el público español ¿cuestiona el modelo televisivo tradicional?

Does the arrival of Netflix in the Spanish audience challenge the traditional television model?

Javier Bustos Díaz

49-61

Generación Z y consumo de información política: entre la televisión y los nuevos formatos mediáticos Generation $Z$ and consumption of political information: between television and new media formats

Ruth Gómez de Travesedo Rojas, Marta Gil Ramírez

$62-79$

Actualidad y comedia: El éxito de El Intermedio y La Resistencia entre los espectadores jóvenes

Information and comedy: The Success of El Intermedio and La Resistencia for young audiences

Inmaculada Concepción Aguilera García

80-95 
El efecto socializador de articular un espacio de comunidad virtual en el ecosistema del aula

The socializing effect of creating a virtual community space in the classroom environment

Lorea Ariadna Ruiz Gómez, Mónica Hinojosa Becerra, Francisco Javier Ruiz San Miguel

Juventude ciberativista e educação: reflexões sobre um jeito hacker de ser

Cyber activity youth and Education: reflections on a hacker way of being

Carla Azevedo de Aragão, Pietro Matheus Bompet Fontoura Alves, Karina Moreira Menezes

La risa grotesca en la obra de Bob Dylan: análisis de la Bacanal y el Festín Carnavalesco en la trilogía folk-rock eléctrica

Grotesque Laughter in Bob Dylan's Work: Analysis of The Bacchanal and the Carnival Feast in the Electric Folk-rock Trilogy

Jesús Albarrán Ligero

El papel del intertexto en el videojuego. Una partida, mil y una historias

Intertext in video games. A game, thousand and one stories

Rocío Serna-Rodrigo

145-158

\section{ÁmBitos PERSONALES PERSONAL ÁmBITOS}

El auge de Twitch: nuevas ofertas audiovisuales y cambios del consumo televisivo entre la audiencia juvenil The rise of Twitch: New audiovisual offers and the transformation of Television consumption among young audiences

\section{ARTÍCULOS ARTICLES}

La recepción de los medios de comunicación para residentes rusos en la Costa del Sol

Mass media reception by Russian residents on the Costa del Sol

Natalia Meléndez Malavé, José Carlos Pozo García

176-192

La calidad periodística en la cobertura de terremotos: Caso Ecuador

Journalistic quality in earthquake coverage: The case of Ecuador

Juan Pablo Arrobo-Agila, Mendoza María, Ignacio Aguaded

La credibilidad publicitaria en la nueva esfera mediática: los universitarios y los medios

Advertising credibility in the new media sphere: university students and the media 
Arco iris en medios brasileños: percepciones sociales sobre una campaña publicitaria LGBTQIA+

Rainbow in Brazilian media: social perceptions of an LGBTQIA+ advertising campaign

Leonardo Ferreira Batista, José Geraldo de Araújo Ramalho Filho

224-239

Rituales y comunicación política: la toma de posesión de los presidentes autonómicos españoles

Rituals and political communication: the Spanish autonomous Presidents inauguration

Ricardo Domínguez García

240-258

RESEÑAS REVIEWS

Una mirada diferente para hacer periodismo

A different look for making journalism

Guillermo Paredes-Otero

259-261

Periodismo y tecnología: una simbiosis continua

Journalism and technology: a continuous symbiosis

Luisa Graciela Aramburú Moncada

262-265

Una propuesta metodológica para impartir la asignatura de redacción periodística

Journalism and technology: a continuous symbiosis

Noelia Ruiz-Alba

266-268 


\title{
El auge de Twitch: nuevas ofertas audiovisuales y cambios del consumo televisivo entre la audiencia juvenil ${ }^{1}$
}

\author{
The rise of Twitch: New audiovisual offers and the transformation of \\ Television consumption among young audiences
}

\author{
Juan Francisco Gutiérrez Lozano \\ Universidad de Málaga | Calle de León Tolstoi, s/n, 29010 Málaga | España | \\ https://orcid.org/0000-0001-6572-135X | jfg@uma.es \\ Antonio Cuartero \\ Universidad de Málaga | Calle de León Tolstoi, s/n, 29010 Málaga | España | \\ https://orcid.org/0000-0001-9099-8254| cuartero@uma.es
}

Fechas | Recepción: 31/05/2020| Aceptación: 18/08/2020 | Publicación final: 15/10/2020

\begin{abstract}
Resumen
La población juvenil española ha modificado en la última década su consumo televisivo, migrando desde los canales tradicionales hacia las nuevas plataformas digitales de diverso contenido. En este fenómeno de alcance global, uno de los sectores audiovisuales que más está creciendo es el de los videojuegos. Twitch, plataforma que ofrece un servicio de vídeo en "streaming", propiedad de Amazon, se ha convertido en el principal soporte audiovisual de contenidos sobre videojuegos entre los públicos más jóvenes. Las emisiones en directo de partidas, comentadas y vividas en grupo por los "streamers" y sus seguidores, aglutinan a millones de espectadores en todo el mundo, como los grandes eventos televisivos. Asimismo, debido al éxito mundial de Twitch,
\end{abstract}

\begin{abstract}
The Spanish youth population has modified its television consumption habits in the last decade, migrating from traditional channels to new digital platforms with diverse content. In this global phenomenon, one of the audiovisual sectors that is growing is the video games industry. Twitch, a platform that offers a streaming video service, owned by Amazon, has become the main audiovisual support for video game content among younger audiences. Live broadcasts of games, commented on and experienced as a group by streamers and their followers, attract millions of viewers around the world, like major television events. Likewise, due to the worldwide success of Twitch, this platform has recently opened, as in its beginnings, to other
\end{abstract}

Forma de citar:

Gutiérrez Lozano, J. F., Cuartero, A. (2020). El auge de Twitch: nuevas ofertas audiovisuales y cambios del consumo televisivo entre la audiencia juvenil ${ }^{1}$. Ámbitos. Revista Internacional de Comunicación 50. pp. 159-175. doi:

10.12795/Ambitos.2020.i50.11 
esta plataforma se ha abierto, como en sus inicios, a contenidos de otro tipo. En ella buscan también acomodo personajes televisivos famosos o procedentes de otras redes sociales como su principal "rival", YouTube. Al tiempo, "otras formas de ver" la televisión o incluso hasta los contenidos radiofónicos son posibles en Twitch, gracias al éxito de sus herramientas de consumo compartido e interacción comunitaria. Este artículo intenta analizar las razones del éxito de Twitch, poniéndolo en relación y contraste con los resultados provenientes de una encuesta realizada a estudiantes universitarios para conocer sus opiniones sobre los contenidos de la televisión en general y los de las nuevas plataformas audiovisuales online.

Palabras clave: Twitch, jóvenes, audiencias, videojuegos, televisión. types of contents. Famous television celebrities or from other social networks such as their main "rival", YouTube are now on Twitch. At the same time, "other ways of watching" television, or even radio content, are also possible on Twitch, thanks to the success of its tools for shared consumption and community interaction. This article tries to analyze the reasons for the success of Twitch, putting it in relation and contrast with the results of a survey carried out with Spanish university students in order to find out their opinions about the content of traditional television and those of the new online audiovisual platforms.

Keywords: Twitch, Youth, Audiences, Videogames, Television.

\section{INTRODUCCIÓN}

Uno de los últimos intentos de Mediaset, compañía líder de la televisión en abierto en España, por congraciarse con los públicos juveniles y atraerlos hacia su plataforma online de pago, Mitele Plus, sacó a la luz de manera paradójica y de nuevo, las vicisitudes a las que se enfrenta el mercado televisivo tradicional a la hora de competir con las nuevas ofertas audiovisuales para estos públicos. La compañía gestó un "reality show" específico para dicha plataforma online, que fue estrenado en septiembre de 2020. Se trató de "Sola/o", consistente en el encierro sucesivo individual de personajes famosos por su aparición en los programas generalistas del grupo emitidos en sus cadenas en abierto, Telecinco y Cuatro.

Pues bien, nada más comenzar la primera de las experiencias, protagonizada por Anabel Pantoja, varios medios de comunicación denunciaron que los contenidos de esta plataforma por suscripción estaban siendo retransmitidos de manera ilegal desde Twitch. Una herramienta originariamente surgida en torno al mundo de los videojuegos, pero donde, además de la piratería de la señal de Mediaset, sus propias características permitían organizar quedadas para ver y comentar en directo estos contenidos televisivos (Vertele, 2020). Algo que ya había ocurrido en 2019 con "Gran Hermano VIP", se repetía ahora con este otro enésimo intento de atraer al público juvenil.

Queda claro que la piratería es un hecho denunciable, pero quizá detrás de esa noticia lo que más sobresalga es la tendencia creciente del público más joven a querer compartir de manera colectiva los visionados de un contenido audiovisual y hacer comentarios y "quedadas" para su consumo simultáneo, compartido y animado mediante las llamadas "Whatch parties". Esta tendencia también se está produciendo a través de otro tipo de plataformas como Discord, una 
red social de chat de voz y vídeo centrada en videojuegos que, entre sus muchas características, permite compartir la pantalla de un usuario, creándose así, de forma privada, reuniones de amigos que a través de esta herramienta de compartir pantalla ven sus programas preferidos de otras plataformas online como Netflix, HBO, Movistar Plus o Amazon Prime. Esta práctica, por ejemplo, ya ha sido asumida por Amazon, que desde septiembre de 2020 activó en su plataforma de vídeos Twitch, precisamente, una función que permite legalmente a sus usuarios de todo el mundo, ya sean productores de contenidos o "streamers", ya sean suscriptores, la posibilidad de ver y comentar juntos el catálogo de películas y series de Amazon, siempre y cuando se acceda previo pago a dicha colección (Alexander, 2020).

Esta nueva forma de "ver televisión" ha venido de la mano, no lo olvidemos, del mundo de los videojuegos. La popularidad y el éxito masivo de contenidos audiovisuales en los que un espectador observa cómo un jugador o varios juegan una partida en directo ha sido el germen de un producto con entidad propia y gran atractivo para los públicos juveniles. Este tipo de retransmisiones, comúnmente denominadas como "gameplay en directo", no es otra cosa sino un visionado colectivo de las hazañas de un "streamer" o jugador.

A partir de esta sencilla idea, sin embargo, tanto las posibilidades tecnológicas asociadas a estas transmisiones digitales, de una parte, como la espectacularización obvia de los grandes campeonatos de videojuegos, por otro lado, han hecho que los conocidos como deportes electrónicos (e-sports), hayan provocado un cambio de paradigma en la forma en la que la juventud disfruta y participa de este consumo de videojuegos como un producto netamente audiovisual. Asimismo, su éxito ha generado una inmensa popularidad de las ventanas tecnológicas que lo hacen posible, y que además brindan posibilidades interactivas que son muy aplaudidas por sus públicos, que ahora parecen extenderse a otros contenidos audiovisuales, incluso hasta los radiofónicos (véase "Twitch: ¿un nuevo canal para la radio?", Espinosa de los Monteros, 2020).

En este sentido, la plataforma de vídeo en directo Twitch, desde 2014 propiedad de Amazon tras una compra valorada en mil millones de dólares, se ha erigido como una de las principales aglutinadoras mundiales de este nuevo tipo de consumo audiovisual. Twitch.tv, como también se conoce a la plataforma, supone un escaparate codiciado, por el número de sus seguidores, para que las empresas de videojuegos o software anuncien en ella sus productos. Aunque ahora, y cada vez más, todo tipo de empresas relacionadas con la tecnología en general (compañías telefónicas o empresas de periféricos para ordenador) u otros productos (ropa) se están anunciando en ella. Asimismo, es el canal por el que los principales campeonatos de deportes electrónicos retransmiten sus eventos. Pero, y no es detalle menor, es un espacio donde muchos usuarios anónimos intentan ganarse la vida emitiendo sus partidas en directo y se convierten en estrellas. De esta manera, los conceptos de "streaming" (como producción de contenidos en directo) y "streamer" (como la persona que lleva a cabo esta producción) adquieren un significado propio si se los asocia específicamente con esta plataforma (Pires \& Simon, 2015).

Desde su aparición en 2011, Twitch logró pronto convertirse en lugar de referencia en lo que se refiere a los videojuegos y a cualquier acontecimiento que derive de este producto cultural. 
Aunque el dominio numérico de YouTube como plataforma web de repositorio y consumo de vídeos a nivel mundial sigue siendo absoluto, Twitch consiguió fortalecer un nicho justo allí donde el gigante de Google no estaba presente: en la transmisión en directo de partidas de videojuegos.

Con el propósito principal de suplir ese déficit, Twitch surgió en 2011 como un subproducto o división de la plataforma de "streaming" de contenidos generalistas Justin.TV. Además de mejorar la calidad y la estabilidad de la emisión en este tipo de plataformas, el número de usuarios dos años después de su aparición, en 2013, ya alcanzaba los 200.000 espectadores al día (TwitchTracker, 2020). Ello propició que en el año 2014 Amazon comprase Twitch (Wingfield, 2014). Hoy se ha convertido en la plataforma de referencia en el mundo de los videojuegos, pero no solo. De esta forma, según el portal Twitch Tracker (2020), el crecimiento de Twitch en la última década ha sido constante, pasando de esos 200.000 espectadores diarios a los 2 millones que tiene actualmente.

En 2020 la plataforma de Amazon puede presumir, además de un seguimiento masivo diario, de contar con cinco millones y medio de usuarios habituales que emiten contenido en directo a través de 76.000 canales en directo (Twitchtracker, 2020). Las cifras que acumula son incuestionables: desde 2017, por ejemplo, la plataforma acumula más usuarios y visitas que otras plataformas digitales como Netflix y HBO (Gómez, 2017) y actualmente se ha convertido en una amenaza para grandes corporaciones como Facebook, Microsoft o Twitter, que ansían quitarle parte de la tarta de usuarios que se dan cita en esta plataforma.

De este modo, Facebook lanzó en 2018 "Facebook Gaming", un intento de competir con Twitch; YouTube hizo lo propio con "YouTube Gaming", pero ante el rotundo fracaso finalmente optó por cerrar su web y su aplicación. Por su parte, Microsoft lo intentó lograr con Mixer, una plataforma donde invirtió grandes cantidades de dinero para despuntar en el mismo terreno de la emisión de videojuegos en directo (Khan, 2019).

Pese a su pujanza, Twitch también ha sufrido algunos ligeros vaivenes en su crecimiento, precisamente ocasionados por esta competencia feroz ligada a un mercado en ascenso, que ha provocado no pocos terremotos en el panorama del consumo en línea de videojuegos retransmitidos en directo. Del mismo modo, influye también en ello una de las características singulares de la propia "comunidad" sobre la que gira su éxito: la atracción generada por sus afamados "streamers" más populares. Así, en septiembre de 2019 Twitch sufrió un notable descenso en sus visualizaciones debido al abandono de dos de sus grandes estrellas, que fueron fichadas por Mixer, la plataforma de Microsoft (González, 2019a; Garro, 2019 y Webb 2019). Se trataba de los dos populares "streamers", "Ninja" y "Shroud". "Ninja" es el seudónimo de Richard Tyler Blevins, un jugador profesional de videojuegos estadounidense, quien antes de su marcha a Mixer poseía el canal de Twitch con más seguidores, sumando 14,7 millones espectadores (González, 2019b); por su parte, "Shroud" es el seudónimo de Michael Grzesiek, un exjugador profesional canadiense del juego "Counter Strike".

En todo caso, de la volatilidad del mercado que intenta hacer frente al casi monopolio de Twitch da buena cuenta que Microsoft anunciase el cierre de Mixer en julio de 2020, así como el final 
de los contratos que estableció con estas estrellas como "Ninja" o "Shroud". La estrategia de la empresa fundada por Bill Gates se dirige ahora hacia el conglomerado con otras grandes empresas tecnológicas, de modo que, en junio de 2020, Microsoft anunció su alianza con Facebook Gaming (D’Anastasio, 2020).

Durante los inicios de la epidemia de la Covid-19, y especialmente durante el confinamiento, Twitch batió todos los récords en el número de seguidores, con un total de 34 millones de horas vistas en un día durante el mes de abril a escala mundial. También rompió la barrera de los cuatro millones de espectadores simultáneos (Branco, 2020). Además, algunas emisiones concretas alcanzaron picos de espectadores de 1,7 millones, superados sólo por lo conseguido en "League of Legends World Championship" durante 2019, con picos de 2 millones de espectadores y una media total acumulada de 21,8 millones de espectadores promedio por minuto (Lolesports Staff, 2019)

De esta manera, no sorprende que Twitch también sea el escaparate preferido para la industria del videojuego en general. Tanto grandes como pequeñas compañías utilizan esta plataforma como canal para anunciar sus productos. Así lo hizo la compañía nipona Sony, por ejemplo, para presentar su nueva consola PS5 en junio de 2020, llegando a obtener un millón de espectadores simultáneos a nivel mundial y un total de 7 millones de espectadores entre las diversas plataformas (Twitch, YouTube y Facebook entre otras) según datos de Esports Charts (2020).

Este mismo portal Esports Charts recoge un informe anual (2019) en el que también se puede observar el éxito de la plataforma Twitch en la emisión de los grandes campeonatos de e-sports. Así, canales como el de la desarrolladora Riot Games (que emite los campeonatos del juego "League of Legends") alcanzó los 124 millones de visualizaciones al año a nivel mundial, mientras que la "Overwatch League", el campeonato del videojuego "Overwatch", obtuvo 102 millones. Y sin olvidar, claro, el rotundo éxito de "Fornite", el videojuego creado por Epic Games, que se situó desde su lanzamiento como el contenido más visto dentro de Twitch (González, 2019a).

Otra cuestión clave para el éxito de la plataforma se encuentra en cómo organiza los dividendos a los creadores de estas emisiones en directo de las partidas que practican ante su audiencia. Mientras que YouTube basa la recompensa a estos "emisores" principalmente a través de la publicidad, Twitch optó por "monetizar" su éxito tanto por la publicidad lograda como por el número de suscriptores conseguidos por los "streamers", así como por otras formas de donación que ofrece la plataforma (Hamilton et. al. 2014). Esta diversidad está provocando que muchos "youtubers" se trasladen a esta plataforma con el objetivo de obtener más dividendos. Es decir, Twitch está basada más en un sistema cuasi de "crowdfunding" en el que sus dividendos dependen de los lazos que puedan establecer con su audiencia.

Se trata, por tanto, este fenómeno de las retransmisiones en directo de videojuegos, de un nuevo subproducto audiovisual con el que ninguna otra plataforma de vídeo puede ahora mismo competir, dado el número acumulado de fieles seguidores que aglutina. Además de lo señalado hasta ahora, deben añadirse las posibilidades comunicativas que ofrece la integración de un chat escrito en la emisión en directo, que abren la puerta a una nueva forma de interacción con la audiencia, justo esa en la que ahora los espectadores de contenidos de ficción, no de 
videojuegos, permiten ver una posibilidad enriquecida a su consumo habitual. Pero el chat de Twitch no sólo se queda en la interacción escrita: la posibilidad de incluir memes, iconos personalizados o emoticonos lo transforman en una herramienta personalizable, en la que cada "streamer" establece sus propias reglas convirtiéndose en una subcultura propia por cada canal y o "streamer".

No en vano, los caminos cruzados entre televisión y Twitch ya habían sido abonados antes de que Amazon abriera la puerta al comentario colectivo de sus series. Ejemplos de sinergias anteriores los podemos comprobar, por ejemplo, en el caso de "The Witcher", la serie de Netflix basada en la obra del polaco Andrzej Sapkowski. Esta ficción provocó un auténtico fenómeno con el videojuego "The Witcher III. Wild Hunt" (2015) una obra del estudio polaco CD Projekt Red basada en los textos de Sapkowski. Las visualizaciones de canales que emitieron en directo partidas del juego aumentaron, así como el número de jugadores en la plataforma de juegos Steam, con 50.000 concurrentes durante el estreno de la serie (Brugat, 2019).

Sin embargo, y como señalan entre otros Spilker, Ask y Hansen (2020), a pesar de la enorme popularidad de Twitch en América, Europa y Asia, su implantación obtiene menos resonancia mediática, hasta ahora, que la de otras plataformas como YouTube o Netflix. Y precisamente, quizá el error está en considerarla "simplemente" una plataforma en directo relacionada con los videojuegos, ya que desde 2015 introdujo otras categorías que van ganando terreno. Como veremos más adelante, en el caso español también se está produciendo, con la introducción creciente de "streamers" relacionados con la música, el humor o el entretenimiento audiovisual en general.

\section{ESTADO DE LA CUESTIÓN}

La investigación académica en torno a los hábitos de consumo de los jóvenes españoles se vio incrementada a finales de la primera década de este siglo, justo en los preámbulos de la aparición de Twitch, con una gran proliferación de análisis centrados en los recientes cambios, relacionados en buena medida con la tecnología (Medrano et al., 2007; Tubella, Tabernero y Dwyer, 2008; Frau, Espina y González, 2006; Bernal Triviño 2009; López Vidales et al. 2010). Estos autores mostraron que este grupo de edad se alejaba cada vez más del consumo audiovisual tradicional, especialmente de la televisión. De esta forma, y al igual que sucedía en el mundo occidental, la batalla por obtener la atención de la audiencia juvenil e infantil se trasladó a todas las plataformas de contenidos audiovisuales online. La tecnología, como herramienta imprescindible en la vida cotidiana de los jóvenes, fue objeto de interés por parte de las empresas de desarrollo audiovisual, que ansiaron obtener la atención de este segmento de la población.

El auge de Twitch y de la retransmisión en vivo audiovisual han sido examinados desde diferentes perspectivas. Una de ellas, aunque como veremos no la única asociada a esta plataforma, ha partido desde el área de los estudios sobre videojuegos o "games studies" (Anderson, 2017). Los "games studies" se focalizaron desde el inicio mayoritariamente en los e- 
sports, como por ejemplo a partir de uno de los títulos que forjó los eventos virtuales deportivos, "Starcraft" (Cheung and Huang, 2011). Poco después de su aparición, el servicio ofrecido por Twitch, su infraestructura técnica y su ecosistema de "prosumidores" (los "streamers" y los seguidores), fueron objeto de atención específica del mundo académico, de manera especial en el mundo anglosajón, pero no exclusivamente (ver Hamilton et al., 2014; Kaytoue, Silva, y Cerf, 2012; Pires \& Simon, 2015).

Otras investigaciones, asimismo, se han centrado en el aspecto social que puede alcanzar Twitch a través de la emisión de partidas. Como recogen estas investigaciones, incluso si un "streamer" está jugando a un juego de un solo jugador es capaz de crear un vínculo social y una comunidad con su propia audiencia (Consalvo, 2017). También Gandolfi (2016) analizó la audiencia de Twitch, mostrando asimismo el potencial que tenía esta plataforma en 2016.

Podemos definir Twitch como un medio de emisión de vídeo en directo que combina la emisión audiovisual con un chat en abierto, denominado por sus siglas en inglés IRC ("Internet Relay Chat"). Así Twitch se construye sobre los "gameplays", la participación de la audiencia y la construcción de una comunidad gracias a sus herramientas interactivas. Twitch por tanto es un medio audiovisual que combina dos vías comunicativas al mismo tiempo, la emisión de vídeo junto a una comunicación textual basada en un chat (Hamilton et al., 2014).

Es evidente que la llegada de Twitch ha propiciado una relación diferente con el videojuego por parte de su audiencia: ya no es necesaria una interacción directa con el juego en sí, sino que se puede actuar a través de otro jugador que enseña a través de una retransmisión en vivo su partida. Las razones de esta pasividad de la audiencia al ver a otro jugador tienen varios sentidos: por un lado, puede ser meramente educacional (aprender a ser un mejor jugador); otras veces, por mero entretenimiento, y otras por su carácter social, interactuando con otras personas a través del citado chat (Vosmeer et. al 2016).

Sin duda este aspecto de Twitch, el que fomenta el sentimiento de comunidad, ese que crea el "streamer" cuando emite sus vídeos y a través del uso del chat, se apoya además en otras herramientas, como la inclusión de una cámara en vivo para que los espectadores vean sus reacciones, lo que genera todo un ecosistema interactivo (Hamilton et al., 2014). La complejidad y posibilidades de la plataforma, como se ha comentado en el primer apartado, es tan amplia que ahora los enfoques dedicados a su investigación se dirigen a diferentes asuntos, relacionados con sus potencialidades en el conjunto global de las redes sociales; con la vinculación de su despliegue audiovisual con otros medios de comunicación; con su utilización estratégica a la hora de conformar una comunicación digital de amplio espectro, especialmente en el caso de los "streamers", cuya figura adquiere cada vez más rasgos profesionales, algo especialmente visible en el caso de las estrellas inicialmente desconocidas (sobre estos asuntos véanse, por ejemplo: Woodcock and Johnson, 2019; Bingham, 2020, o Sjöblom, et. al., 2018).

Desde el punto de vista televisivo, quizá el acercamiento más interesante es el recientemente aportado por Spilker, Ask y Hansen (2020), quienes afrontan el complejo significado de las audiencias de Twitch en comparación con la televisión partiendo de un concepto indisociable, referido a la flexibilidad en el consumo de la plataforma practicada por sus seguidores, que 
aportan significados distintos a partir de sus experiencias múltiples en estos canales de la plataforma. Además, subrayan la ironía de que estas emisiones de larga duración en directo se conviertan en su gran polo de atracción, algo que establece paralelismos obvios, salvando las distancias, con la televisión lineal tradicional.

\section{OBJETIVO Y METODOLOGÍA}

Expuestas las líneas generales de eclosión del fenómeno Twitch y de su arraigo entre su cuantioso público, y mencionados algunos acercamientos académicos iniciales sobre su diversa significación e influjo cruzado con los consumos televisivos tradicionales, procedemos ahora a exponer cómo hemos abordado el fenómeno a través de la presente investigación. En concreto, nuestro acercamiento se inserta en un proyecto más amplio que, de una parte, busca conocer las razones del alejamiento de los jóvenes entre 14 y 24 años de la televisión tradicional, así como, de otra, intenta mostrar si ello se explica por el repetido uso de estereotipos negativos de la juventud en la oferta de contenidos televisivos de los canales tradicionales, frente a la posible novedad aportada en este sentido por las nuevas plataformas online.

En este artículo, en concreto, vamos a analizar cómo la plataforma Twitch atrae cada vez más a una cuantiosa audiencia juvenil precisamente gracias al "salto" de muchos creadores de contenidos ("youtubers", "influencers" de redes sociales y hasta estrellas procedentes del ámbito radiofónico o televisivo) a esta plataforma. En concreto, y a partir de un sondeo exploratorio para realizar posteriormente grupos cualitativos y análisis más pormenorizados, quisimos obtener la opinión de jóvenes universitarios españoles sobre sus consumos audiovisuales generales, incidiendo en los objetivos citados relacionados con la televisión tradicional y las nuevas plataformas.

La metodología llevada a cabo para esta herramienta indagatoria preliminar fue la de una encuesta sobre su consumo televisivo actual, tanto de canales de la televisión tradicional como de los contenidos audiovisuales digitales o exclusivamente ofrecidos online (plataformas y redes sociales). Las preguntas se configuraron tanto con respuestas abiertas como cerradas, con el afán inicial de sondear de manera aproximativa las líneas generales de usos por las que luego pudiéramos interrogar a grupos concretos con mayor profundidad. La muestra final fue de 220 estudiantes universitarios, estudiantes de distintos grados oficiales de la Universidad de Málaga, agrupados en distintas edades entre los 19 y 21 años. El 59\% de los informantes fueron mujeres y el $40 \%$ hombres. La encuesta fue realizada en la primavera de 2019.

El cuestionario se constituyó en torno a 50 preguntas divididas en cinco grandes bloques sobre: consumo de la televisión tradicional entre los jóvenes; consumo de la televisión online o VOD en plataformas digitales; el uso de YouTube y otras plataformas; el uso de redes sociales; $y$, por último, la percepción, de la juventud sobre la representación de su grupo de edad tanto en contenidos televisivos de los canales tradicionales de televisión como en las nuevas plataformas. 
Lo singular del caso es que, si bien este estudio concebido como herramienta preliminar no estaba dirigido al conocimiento de Twitch ni de sus canales, en las respuestas recogidas se observó el cruce de inferencias entre unas y otras ventanas de creación y oferta audiovisual. De este modo, en el consumo juvenil declarado se detectan estas pasarelas naturales, donde las estrellas de YouTube o de programas televisivos de cadenas de pago, ya están haciendo presencia y buscando huecos en este otro sitio, hasta ahora no tan conocido, donde las experiencias de los usuarios juveniles encuentran un fácil y atractivo acomodo. Y ello se explica, claro, por el influjo de la "segunda pantalla" en todos los fenómenos de consumo audiovisual de la generación juvenil (Tussey, 2014).

\section{RESULTADOS}

De los datos generales procedentes de la encuesta podemos reseñar, como dato significativo, que los jóvenes encuestados manifestaron seguir consumiendo contenidos televisivos tradicionales en un porcentaje elevado (76\%), si bien un $22 \%$ afirmaba no ver nunca la televisión tradicional en su emisión ordinaria. Ese porcentaje de jóvenes parece haber desconectado por completo de la emisión lineal y realiza su consumo audiovisual principal bien a través de las plataformas online, bien consumiendo contenidos tradicionales en diferido o en los espacios en la web de las cadenas de televisión.

No sorprende por tanto que el receptor tradicional ya no sea el dispositivo preferido para ver la televisión por parte de los jóvenes encuestados. De esta manera, el 35\% sostenía usar el ordenador para ver los contenidos de estos canales, un $25 \%$ el móvil, un $9 \%$ las tabletas, y solo un $14 \%$ hace uso del receptor televisivo, pero mediante una conexión a internet (conectando un ordenador al receptor, con un Chromecast, con Apple TV, etcétera). Se observa así que el ordenador capitaliza las capacidades del receptor tradicional de televisión, pues es mucho más versátil y las principales plataformas funcionan de forma más eficiente a través de este dispositivo. De esta forma, el ordenador ya es el centro del consumo audiovisual entre los más jóvenes, donde pueden saltar desde los contenidos que sube la televisión tradicional a la carta, a las plataformas como Netflix o HBO, o a plataformas de vídeo como YouTube o Twitch. Es cierto que cada vez más televisores ofrecen la versatilidad de su funcionamiento, pero entre los encuestados parecen diferenciar bien, todavía, esta dualidad.

De otro lado, y ligado con lo anterior, el $89 \%$ de los jóvenes encuestados consume contenidos televisivos (series, películas, programas o "reality shows") a través de plataformas de contenido online como pueden ser Netflix, HBO, Amazon Video o Movistar Plus. De entre ellas, el 49\% afirma estar suscrito a Netflix, a la que le sigue la plataforma privada española Movistar Plus (23\%) y en tercer lugar Amazon Prime Video (8\%).

¿Cuál es la razón que lleva a los jóvenes a acudir a estas plataformas para satisfacer su consumo audiovisual? De entre la variedad de respuestas ofrecidas por los encuestados podemos obtener cuatro principales razones. El $22 \%$ señala la variedad de contenidos que ofrecen estas empresas; una razón conectada también con la cantidad de contenidos de los que disponen. La segunda razón que argumentan (el 21\%), es la del entretenimiento: asocian estas plataformas a su forma 
de evasión, de relajarse o de irse a dormir después de un día de trabajo o estudio. La tercera razón, y no menos importante, es la comodidad (17\%), que se traduce en la disponibilidad de contenidos para su consumo personalizado y la ausencia de pausas publicitarias que tienen muchas de estas plataformas (especialmente Netflix y HBO pues Movistar Plus y Amazon sí la incluyen). Finalmente, para el $13 \%$, es la libertad de elección, la falta de ataduras a una programación convencional, lo que determina su predilección por estas plataformas.

En cuanto a la preferencia del dispositivo con el que ven estas plataformas de contenidos audiovisuales, de nuevo el ordenador (35\%) es el dispositivo preferido, seguido de la televisión (26\%) usando una conexión de Internet a través del propio equipo u otro dispositivo. Las razones que señalan para hacer uso principalmente del ordenador y de la televisión a través de una conexión es la comodidad (44\%), seguido del tamaño mayor de la pantalla frente a otros dispositivos como el móvil o la tablet.

Posiblemente estos datos muestran el importante cambio mental que ha supuesto la llegada masiva de la televisión online o en "streaming" (usado aquí con un sentido distinto al de Twitch, pero confluyente). Ahora es el consumidor quien tiene la capacidad de elección, quien decide, quien para, retrocede o inicia el contenido que quiere ver a su gusto. Una vez habituado a este hábito es complejo volver a la dinámica televisiva tradicional, de ahí la mutación que se está provocando entre los más jóvenes, que poco a poco se alejan de la televisión tradicional. No obstante, también hay un cambio en cierta medida negativo en el modo de ver así la televisión, y es que generalmente se produce de forma solitaria o privada, mientras que la televisión tradicional y su consumo habitual era una costumbre familiar, que se producía generalmente en el salón de casa. Sin embargo, y como ahora veremos, existen algunos elementos en el consumo actual de plataformas de vídeo que entrelazan paradójicamente los modos de la "vieja" televisión con las posibilidades que ofrece Twitch y, entre otros aspectos, destaca el seguimiento de retransmisiones síncronas o la relevancia que en ella adquieren los personajes, protagonistas o "estrellas" de sus emisiones, como ocurre en los canales tradicionales televisivos.

\subsection{La paradoja de Twitch: el éxito de las figuras televisivas y de YouTube en sus contenidos síncronos}

Como hemos dicho, pese al evidente auge de Twitch, en determinados aspectos aún no puede ensombrecer el predominio de YouTube, que sigue siendo la principal plataforma de vídeos online. Siguiendo con los resultados de la encuesta realizada a jóvenes universitarios, un $95 \%$ de los mismos declara que consume contenidos de esta plataforma de Google. Y, además, de manera intensiva: un $45 \%$ lo hace todos los días y un $26 \%$ casi todos los días.

En el caso de los dispositivos utilizados para este consumo, se detecta un gran cambio en YouTube con respecto a la televisión, pues en este caso el 53\% de los encuestados prefiere ver sus vídeos en el teléfono móvil, seguido de un $29 \%$, que prefiere el ordenador. Es evidente que para las plataformas en "streaming" la comodidad del ordenador y la televisión se impone, mientras que en YouTube predomina la inmediatez y el acceso rápido a través del móvil. 
Los contenidos mayoritarios que los jóvenes encuestados declaran seguir a través de YouTube también divergen en cierta medida de los referidos a la televisión tradicional o la televisión online que consumen. Si bien el entretenimiento (45\%) es el principal tipo de contenidos consumido (dato coincidente con el de otras alternativas audiovisuales), en el segundo lugar se sitúa la música (44\%), luego el humor (27\%), seguido en cuarta posición por la información (17\%). El humor y la música son dos tipos de contenidos que tienen un gran impacto y suponen uno de los mayores atractivos para los jóvenes en esta plataforma de vídeos. Pero también es importante reseñar la quinta posición que ocupan los videojuegos (11\%). Se entiende así que el público fiel a estos últimos contenidos obtenga en Twitch su principal oferta especializada, dotada además de características de añadido atractivo a las de YouTube.

En este sentido, como apuntábamos, la clave del auge de Twitch la encontramos asimismo en la fama que obtienen sus creadores de contenido. En efecto, se convierten en elementos o figuras de "arrastre" de la audiencia. Lo curioso es que, a partir de los datos de la encuesta, observamos en las prácticas de consumo audiovisual de la juventud universitaria encuestada algunos datos cruzados que dan buenas pistas de cómo se entrelazan sus seguimientos tanto en la televisión tradicional (ya sea en canales generalistas o de pago), las redes sociales y las plataformas de vídeo como YouTube o la propia Twitch.

Veamos por ejemplo el caso de sus espacios televisivos preferidos. Cuando se interroga a los jóvenes por los programas de televisión preferidos el primer lugar lo ocupa "La Resistencia" (\#0, de Movistar Plus), que presenta el humorista David Broncano. El impacto de este presentador entre los más jóvenes es palpable. Además, y pese a emitirse su formato en un canal de pago, la apuesta del programa de Movistar Plus por subir gratuitamente las entrevistas del espacio a YouTube son todo un éxito en cuanto a número de seguidores y visionado.

En estas entrevistas predomina un estilo desenfadado, con mucho humor y una gran naturalidad. Una conexión que convierte a David Broncano en un personaje de relevancia con tirón exportable a otras ventanas audiovisuales. En este sentido, por ejemplo, y coincidiendo con el confinamiento provocado por la pandemia de la Covid-19, David Broncano comenzó a participar en Twitch junto a algunos "streamers" españoles como Ibai Llanos, mediante su participación activa en partidas de diversos videojuegos como "Valorant" (Riot Games), atrayendo una media de 50.000 espectadores. A raíz de esta inserción en la plataforma de Amazon, Broncano creó su propio canal en Twitch ("Broncanogamer"), que en muy poco tiempo consiguió superar los 90.000 seguidores (junio de 2020). En su primera emisión, el 14 de junio de 2020, este canal obtuvo picos de 12.000 espectadores simultáneos. De esta forma, puede observarse cómo una figura de los medios "tradicionales" (recordemos que la fama de Broncano, antes de su paso por la televisión, provenía de un espacio de radio, aupado por sus emisiones en YouTube y podcasts, titulado "La vida moderna" y emitido por la Cadena SER), puede "mover" a su audiencia desde plataformas más consolidadas hacia nuevas ventanas como Twitch, gracias sobre todo a la capacidad de estos creadores de contenidos para conectar con el público juvenil. 
Siguiendo con los resultados de la encuesta, no sorprende tampoco que cuando se les pregunta a los jóvenes por sus canales preferidos en YouTube, y tras "La Resistencia", el segundo "youtuber", "influencer" o creador de contenidos audiovisual preferido por los encuestados sea Auronplay. Se trata de uno los "youtubers" más importantes a nivel nacional: con 16 millones de suscriptores, es el tercero más seguido en español. En Twitch tiene asimismo su propio canal y es el segundo "streamer" más seguido en España, con 3,4 millones de fieles tras El Rubius que cuenta con 5 millones (Fuente: Sullygnome).

Auronplay es el seudónimo de Raúl Álvarez Genes, joven de Badalona que ganó su popularidad gracias, en sus inicios, a los vídeos en YouTube donde vertía críticas hacia otros "youtubers", aunque actualmente se centre sobre todo en la difusión de bromas telefónicas o análisis de vídeos humorísticos. El tono de este "youtuber" es directo y crítico, y no tiene reparos en tratar temas polémicos. Sin embargo, es en su nueva aventura en Twitch donde Auronplay se está convirtiendo en todo un referente para el público juvenil, con una media diaria de 100.000 personas siguiendo su contenido, que se dedica en especial a realizar "roleplay" dentro del videojuego "Gran Theft Auto V" (GTA V) . El "roleplay" es un subgénero en sí mismo, que consiste en un juego de roles entre diversos usuarios dentro del juego GTA V. La serie que lleva a cabo AuronPlay de sus aventuras en esta serie GTA V, dentro de esta dinámica del "roleplay" lo está convirtiendo en toda una referencia de este canal (Merino, 2020).

El caso de Auronplay es un ejemplo característico de cómo se está produciendo este trasvase no solo de figuras con tirón o algunos anclajes en el ámbito mediático más tradicional (como pueda ser Broncano), sino también entre "youtubers" que nacieron en el medio digital. Así, es evidente que muchos creadores de contenidos que producían todo su contenido en YouTube empiecen a mudarse a Twitch.

Otro caso paradigmático es el de Alexelcapo, seudónimo de Alejandro Fernández Miret, "youtuber" y "streamer" mallorquín, que en los resultados de nuestra encuesta aparece asimismo como el quinto personaje preferido por los encuestados de entre los creadores de YouTube. Precisamente, Alexelcapo ha manifestado en numerosas ocasiones su estrategia para la creación de contenidos: primero realiza toda su práctica audiovisual en Twitch, emisión que luego recorta y "resube" a YouTube. De esta forma, y a partir de un mismo contenido audiovisual, este creador es capaz de sacar partido económico doble al mismo producto audiovisual. Esta práctica es asimismo seguida por otros creadores de gran fama en ambas plataformas, YouTube y Twitch, como AuronPlay o El Rubius, en una lógica intención de obtener más beneficios. Los cambios recientes en la política de YouTube en relación con la publicidad y la pérdida de ciertos anunciantes han provocado un descenso de los ingresos que perciben estos creadores, que ahora ven en Twitch una ventana de oportunidad indudable (Sierra, 2019).

De este modo, una figura asociada al éxito de YouTube entre la juventud española como El Rubius anunció en 2018 su paso a Twitch, aunque aclaró que no dejaría de subir contenidos a la plataforma que le había granjeado su popularidad (Galeano, 2018). Un movimiento que ha sido todo un éxito, según los datos que proporciona la plataforma "Sullygnome", web centrada en las estadísticas y análisis de Twitch y de los creadores de contenidos más exitosos. En el periodo 
de marzo a septiembre de 2020, en España el primer puesto en la plataforma de Amazon lo obtuvo el streamer "Auronplay", con 37 millones de horas reproducidas y una media de espectadores de 80.000 cada día. A este le siguió, "El Rubius" con 34 millones de horas de reproducción y 61.000 espectadores; "TheGrefg" fue el tercero con un total de 29 millones de horas reproducidas y 43.000 espectadores de media, y luego se encontraba Ibai Llanos, quien tras su fichaje por G2 Esports en 2020, comenzó a crear contenido a tiempo completo, consiguiendo 29 millones de horas de reproducción y una media de 31.000 personas siguiéndole a diario (Sullygnome, 2020).

De esta manera, muchos de los citados por los encuestados como sus creadores preferidos de canales de YouTube tienen ahora su traslación en Twitch. Pero llama la atención, en todo caso, que algunos de los "youtubers" con más seguidores y conocidos en España apenas sean mencionados por estos jóvenes universitarios (caso, por ejemplo, del propio El Rubius, Wismichu, o incluso del segundo canal más seguido de mundo, PewDiePie, que también tiene una presencia anecdótica entre los resultados de la encuesta).

Probablemente entre las predilecciones de la juventud dentro de estas plataformas, ya sea YouTube o Twitch, el perfil concreto de edad de los jóvenes tienda a elegir a unos determinados creadores de contenido como referencia frente a otros. Así, podemos llegar a plantear la hipótesis, por confirmar en pasos posteriores de la investigación, de que creadores de contenidos como El Rubius atraigan a los jóvenes de menor edad, mientras que los de edad universitaria se sientan más atraídos por creadores de contenidos como Auronplay, Broncano o Alexelcapo, en los que prima una gran naturalidad y un fuerte componente humorístico.

\section{DISCUSIÓN Y CONCLUSIONES}

A tenor de lo visto en los apartados anteriores, el auge de Twitch se incardina en un cambio de hábitos del consumo audiovisual de la juventud mundial, y por ende también de la española. La mutación de este panorama hace que existan vasos comunicantes entre todas las ventanas de creación y oferta audiovisual. De esta manera, los formatos famosos en YouTube, o con ciertas ramificaciones en los medios más cercanos al "mainstream" (como los ejemplos citados de David Broncano; o hasta los contenidos pirateados de cadenas tradicionales), encuentran su traslación ahora en Twitch, lugar donde la juventud parece hallar un espacio de interacción que refuerza su sentido de comunidad o de audiencia, donde encuentran contenidos de su gusto y que está naturalmente asociado a experiencias anteriores. $\mathrm{Y}$, aunque el origen y su éxito radiquen obviamente en los contenidos ligados al videojuego, su impacto y su proyección actual permiten augurar un desarrollo futuro no necesariamente asociado en exclusiva a este género de retransmisión en directo de partidas, como la propia Amazon está favoreciendo en sus "fiestas" de visionado conjunto de Amazon Prime Video.

Por tanto, podemos considerar que Twitch no evidencia solamente un cambio de tendencia en cuanto al consumo audiovisual de los jóvenes, sino que provoca una transformación más amplia en la forma en la que esta audiencia se relaciona con un producto audiovisual. La participación directa de la juventud a través del chat (IRC), de las donaciones o de las suscripciones, ofrece 
una oportunidad directa de poder interactuar, hablar y crear una comunidad en torno a las figuras de los creadores de contenidos. Este hecho es sin duda el elemento más innovador, si bien el propio desarrollo de la plataforma sugiere concomitancias con la "antigua" fórmula de éxito de la comunicación televisiva: la audiencia en directo, compartiendo un mismo contenido de manera síncrona, fue sin duda el éxito que aupó al medio televisivo; salvando las distancias tecnológicas, ese germen también está presente en Twitch.

Aunque YouTube siga teniendo un papel esencial, se detecta una migración evidente de los creadores de contenidos de la plataforma de Google hacia Twitch, con el fin mencionado de obtener más beneficios y de hacer crecer a su audiencia potencial. De este modo, YouTube se convierte por un lado en un enlace que sirve como expositor de los contenidos, como una plataforma que aglutina los mejores o más interesantes vídeos. Mientras, Twitch se eleva como la plataforma preferida para la propia creación de contenidos, ligada especialmente al directo. De esta manera, YouTube parece hacer las veces de repositorio en diferido, mientras que Twitch se reserva como la ventana privilegiada para la emisión en directo.

No cabe duda, además, de que Twitch es asimismo el espacio por excelencia de los grandes eventos deportivos online. La gran mayoría de los grandes campeonatos se emiten a través de esta plataforma, que es utilizada por las empresas desarrolladoras de software y hardware de videojuegos, así como para difundir cualquier anuncio relacionado con estos productos. Pese al gran desembolso que están realizando empresas competidoras como Google o Microsoft para el desarrollo de plataformas de contenidos en directo, Twitch, propiedad de Amazon, sigue liderando el mercado de visualización de productos audiovisuales sobre videojuegos. De ahí los esfuerzos por plantarle cara, la reciente clausura de Mixer, de Microsoft, o la fusión de esta plataforma con Facebook Gaming.

Pero además de esta conformación comunitaria global de un espacio mediático en torno a los videojuegos, Twitch es mucho más, y lo fue casi desde el principio. También es un espacio audiovisual de producción y emisión de contenidos audiovisuales específicos, que propicia la interacción con la audiencia de manera natural. Aquí también es esencial entender que las comunidades que se originan en torno a los "streamers" en Twitch poseen hasta una jerga concreta que hay que dominar para que se produzca la interacción, pero los niveles de participación de los usuarios también se definen por sus propias cualidades o posibilidades.

Como se ha visto a partir de la aproximación al consumo audiovisual de jóvenes universitarios, en Twitch sobresale el papel del ordenador personal como dispositivo de consumo es esencial para poder entender su relación con las audiencias, si bien el desarrollo de aplicaciones móviles mejor dispuestas podrá matizar esta preponderancia. Sin embargo, el ordenador permite un uso secundario de las emisiones, por lo que, para las audiencias, además de ser más fácil poder participar e interactuar en el chat, pueden tener estas retransmisiones como complemento de fondo mientras realizan otras actividades. Esta es una característica de Twitch que, por ejemplo, se vislumbra como una vía de expansión posible de los contenidos radiofónicos.

La pandemia de la Covid-19 también ha tenido un efecto potenciador en el caso de Twitch en España, especialmente por la llegada de nuevas figuras audiovisuales a esta plataforma. Es el 
caso de figuras como la de David Broncano o la de Jaime Altozano, youtuber y productor musical, quienes auguran una diversificación mayor de los contenidos audiovisuales futuros de esta plataforma de gran seguimiento entre las audiencias juveniles. Pronto, quizá, no sea vista como la plataforma donde las prácticas ilegales fecundan, sino donde los proyectos audiovisuales y televisivos más innovadores puedan encontrar su sitio.

\section{Referencias}

Alexander, J. (2020). "Twitch now lets anyone host an online movie party with the Amazon Prime Video library", The Verge, 2 de septiembre de 2020. Recuperado de: https://cutt.ly/Xf9plib

Anderson, S. L. (2017). Watching people is not a game: Interactive online corporeality, Twitch. tv and videogame streams. Game Studies, 17(1), 1-16.

Bernal, A. I. (2009). Los nuevos medios de comunicación y los jóvenes. Aproximación a un modelo ideal de medio. Bruselas: Euroeditions.

Bingham, C. M. (2020). Talking about Twitch: Dropped Frames and a normative theory of new media production. Convergence, 26(2), 269-286. Doi: https://doi.org/10.1177/1354856517736974

Branco, Alex, (2020). La otra cara de la cuarentena: los 'streamers' baten récords de audiencia. El Español. 16 de abril. Recuperado de: https://cutt.ly/df9pjfi

Brugat, M. (2019). Miles de jugadores vuelven a The Witcher 3 gracias a la serie de Netflix. La Vanguardia, 24 de diciembre. Recuperado de: https://cutt.ly/Zf9pgq7

Cheung, G., y Huang, J. (2011). Starcraft from the stands: Understanding the game spectator. CHI '11 Proceedings of the 2011 Annual Conference on Human Factors in Computing Systems, 763-772. Doi: https://doi.org/10.1145/1978942.1979053

Consalvo, M. (2017). Player one, playing with others virtually: What's next in game and player studies. Critical Studies in Media Communication, 34 (1), 84-87. Doi: https://doi.org/10.1080/15295036.2016.1266682

D'Anastasio, C. (2020). "Microsfot Gives up on Mixer". Wired, 22 de junio. Recuperado de: https://cutt.ly/Ef9pd2G

Espinosa de los Monteros, M.J. (2020) "Twitch: ¿un nuevo canal para la radio?". El País, 10 de septiembre de 2020. Recuperado de: https://cutt.ly/if9pdqs

Esports Charts (2020). Tournaments statistcs PS5 Future of Gaming Show. Esports Charts. Recuperado de: https://cutt.ly/5f9ppmi

Esports Charts (2019). Viewership Statiscs of 2019 Esports \& Streaming. Esports Charts. Recuperado de: https://escharts.com/2019

Frau Marhuenda, C.; Espinar Ruiz, E. y González Río, M. J. (2006). Diferencias entre pantallas. Pérdida de importancia de la televisión como medio de comunicación entre los jóvenes españoles. En XXI Congreso Internacional de Comunicación CICOM: Los jóvenes y el nuevo escenario de la comunicación. Pamplona: Facultad de Comunicación, Universi-dad de Navarra.

Galeano, S. (2018). El Rubius se pasa a Twitch: el mayor youtuber español se acerca a la plataforma de video de Amazon. Marketingk4ecommence, 9 de noviembre. Recuperado de: https://cutt.ly/nf9pi24

Gandolfi, E. (2016). To watch or to play, it is in the game: The game culture on Twitch. tv among performers, plays and audiences. Journal of Gaming \& Virtual Worlds, 8(1), 63-82. Doi: https://doi.org/10.1386/igvw.8.1.63 1 
Garro, J. (2019). La millonaria guerra de las plataformas por tu streamer favorito: Ninja a Mixer, Lolito a Facebook y Grefg a Twitch protagonizan el mercado de fichajes. Xataka, 15 de noviembre. Recuperado de: https://cutt.ly/zf9ptBP

Gómez, M. (2017). "Twitch acumula más usuarios y visitas que Netflix y HBO". 3Djuegos, 20 de octubre. Recuperado de: https://cutt.ly/Of9prl0

González, S. (2019a). Los líderes del 'streaming': de Fornite a Twitch, el videojuego reina en Internet. EI País, 10 de noviembre. Recuperado de: https://elpais.com/tecnologia/2019/11/05/actualidad/1572945893 362754.html

González, S. (2019b). Twitch, Mixer y el fichaje de 'Ninja': así es la pelea de las grandes plataformas de videojuegos. El País, 16 de agosto. Recuperado de: (González, 2019) https://cutt.ly/rf9o8jg

Hamilton, W. A., Garretson, O., \& Kerne, A. (2014). Streaming on twitch: fostering participatory communities of play within live mixed media. En Proceedings of the SIGCHI conference on human factors in computing systems, pp. 1315-1324. Doi: https://doi.org/10.1145/2556288.2557048

Kaytoue, M., Silva, A., \& Cerf, L. (2012). Watch me playing, I am a professional: A first study on video game live streaming. En Proceedings of the 21st international conference companion on world wide web, pp. 1181-1188. https://doi.org/10.1145/2187980.2188259.

Khan, I. (2019). Why Twitch is Still the King of Live Game Streaming. New York Times. 15 de diciembre. Recuperado de: https://cutt.ly/Kf9oN7M

Lolesports Staff. (2019). 2019 World Championship hits record viewership. League of Legends. Recuperado de: https://cutt.ly/nf9oCvJ

López Vidales, N. et al. (2010). Preferencia juvenil en nuevos formatos de televisión: tendencias de consumo en jóvenes de 14 a 25 años. Observatorio del Ocio y del entretenimiento Digital.

Medrano, C. y Palacios, S.; Barandiaran, A. (2007). Los hábitos y preferencias televisivas en jóvenes y adolescentes: Un estudio realizado en el País Vasco. Revista Latina de Comunicación Social, 62, pp. 13-27.

Merino, A. (2020). Quedada en un cine de GTA V para ver la serie de roleplay de Auron. eSports (As.com). 27 de mayo. Recuperado de: https://cutt.ly/Yf9oLG5

Pires, K., \& Simon, G. (2015, March). YouTube live and Twitch: a tour of user-generated live streaming systems. In Proceedings of the 6th ACM multimedia systems conference, pp. 225-230. Doi: https://doi.org/10.1145/2713168.2713195

Sierra, I. (2019). Cómo cambiará YouTube en 2020: multas, contenido infantil y un 90\% menos de ingresos para los youtubers". Xataka. Recuperado de: https://cutt.ly/if9oJOE

Sjöblom, M.; Törhönen, M.; Hamari, J.; Macey, J. (2018). The ingredients of Twitch streaming: Affordances of game streams. Computers in Human Behavior, vol. 92, p. 20-28. Doi: https://doi.org/10.1016/i.chb.2018.10.012

Spilker, H.S; Ask, K. y Hansen, M. (2020). The New Practices and Infrastructures of Participation: How the Popularity of Twitch.tv challenges old and new Ideas about Television Viewing. Information, Communication \& Society, 23: 4. Doi: https://doi.org/10.1080/1369118X.2018.1529193

SullyGnome (2020). Twitch channels with the highest peak viewership - stats and analytics. SullyGnome. Recuperado de: https://cutt.ly/df9oDAD

Tubella, I.; Tabernero, C. y Dwyer, V. (2008). Internet y Televisión. La guerra de las pantallas, Barcelona, Ariel.

Tussey, E. (2014), Connected Viewing on the Second Screen: The Limitations of the Living Room. En: Connected Viewing: Selling, Streaming, \& Sharing in the Digital Era, Holt, J., Sanson, K. (Eds.). New York: Routledge, pp. 202-216. 
TwitchTracker (2020). Twitch Statitcs \& Charts. TwitchTracker. Recuperado de: https://twitchtracker.com/statistics

Vertele (2020). Espectadores de Mediaset recurren a la piratería para ver 'Sola' de forma ilegal. Vertele, 17 de septiembre 2020. Recuperado de: https://cutt.ly/wf9oPGA

Vosmeer, M., Ferri, G., Schouten, B., y Rank, S. (2016). Changing roles in gaming: twitch and new gaming audiences. En DiGRA/FDG 2016-Abstract proceedings of the first international joint conference of DiGRA and FDG, Dundee, Scotland.

Webb, K. (2019). Ninja ditching Twitch for Microsft's Mixer was a brilliant decision. Business Insider, 4 de Agosto de 2019. Recuperado de: https://cutt.ly/mf9oOpu

Wingfield, N. (2014). What's Twitch? Gamers Know, and Amazon is Spending \$1 Billion on it. New York Times, 25 de Agosto. Recuperado de: https://cutt.ly/Gf9oYWn

Woodcock, J. y Johnson, M. R. (2019). Live Streamers on Twitch.tv as Social Media Influencers: Chances and Challenges for Strategic Communication. International Journal of Strategic Communication, 13(4): 321-335. Doi: https://doi.org/10.1080/1553118X.2019.1630412

\section{Notas}

${ }^{1}$ Esta investigación forma parte de los resultados del Proyecto de I+D CSO2017-85483-R "JUVEN-TV. Nuevos consumos frente a viejos estereotipos: análisis de la recepción por parte de la juventud española de sus representaciones televisivas actuales", financiado por el Ministerio de Ciencia, Innovación y Universidades. 